\title{
Use of Taus in ATLAS
}

\author{
Ian Hinchliffe $\mathrm{a}^{\text {* }}$ \\ ${ }^{a}$ Lawrence Berkeley National Laboratory, Berkeley, CA 94720 USA
}

At the LHC, new particles can be expected that decay to final states involving taus. Examples are given from simulations by the ATLAS experiment showing how such final states can be exploited.

\section{INTRODUCTION}

The total production rate for taus at a hadron collider is not a useful quantity. Taus must have significant transverse momentum $\left(p_{T}\right)$ in order to be observable. Leptonic decays of taus will yield isolated electrons or muons that can be detected but these can also be directly produced so discriminating their origin can be difficult. Hadronic decays of taus result in jets that must be distinguished from jets arising from QCD processes using the particle multiplicity and invariant mass.

\section{PROPERTIES OF TAUS AT LHC}

The dominant standard model production that results in an observable sample is $W \rightarrow \tau \nu$ which produces $\sim 1.5 \times 10^{8}$ events per $10 \mathrm{fb}^{-1}$. Given this large sample it is reasonable to ask if any useful measurements of tau properties can be made. Measurement of the lifetime is difficult as it requires a determination of the decay length using the vertex tracking system and knowledge of the tau momentum. In the case of $Z \rightarrow \tau \tau$ the momentum can be reconstructed using a constrained fit involving the $Z$ mass (see Section 3). The process is statistics limited to a precision of approximately 1.8 fs for $30 \mathrm{fb}^{-1}$ of data ([1] vol I p. 305). Use of the $W$ decay which has a much larger rate is more difficult as there are two neutrinos [2]. Two methods were attempted involving using the observed tau decay products and the tau mass constraint to determine the momentum

\footnotetext{
*This work was supported in part by the Director, Office of Science, Office of Basic Energy Research, Division of High Energy Physics of the U.S. Department of Energy under Contract DE-AC03-76SF00098
}

of the neutrino in the tau decay and an estimator method [3]. In this case the statistical error could be less due to the larger event sample provided that the QCD background can be rejected. However, the systematic uncertainties from alignment and other sources are difficult to estimate.

Rate decays of the tau can provide a probe of new physics. Lepton number is known to be violated in the neutrino sector and the rare decays $\tau \rightarrow \mu \gamma, \tau \rightarrow 3 \mu$ or $\tau \rightarrow \mu e^{+} e^{-}$can be expected to occur. In many models [4] [5], [6] the first of these is expected to be the largest and a simulation will be discussed here [7]. The signal is an isolated $\mu$ and photon whose invariant mass reconstructs to the tau. There are two sources of background $\tau \rightarrow \mu \nu \nu \gamma$ and $W \rightarrow \gamma \tau \rightarrow \gamma \mu \nu \nu$; the latter dominates. After cuts to reduce the background, the signal acceptance is approximately $0.5 \%$ and the mass resolution is $20 \mathrm{MeV}$ resulting in a background of approximately 17 events per $10 \mathrm{fb}^{-1}$. The resulting limit is not competitive with what is possible at Belle and BaBar unless the full luminosity of the LHC can be exploited. A study in this environment where the pile-up of minimum bias events degrades resolution and increases background has not been undertaken.

\section{RECONSTRUCTION OF $Z \rightarrow \tau \tau$}

The $H \rightarrow \tau \tau$ process is important as a tool for searching for Higgs bosons at LHC. As a prelude to this and to illustrate the technique, I will first discuss $Z \rightarrow \tau \tau$. Missing neutrinos imply that tau energy cannot be measured directly. However the direction of tau can be obtained from observed decay products as the energy of the pro- 
duced taus is large compared to the mass. If $Z$ has signifificant transverse momentum so that tau's are not back to back in phi and the only missing $P_{T}$ in the event arises from tau decay, then the tau momenta and invariant mass of the $\tau \tau$ system can be reconstructed by assuming that the neutrinos follow the direction of the observed decay products. These events have no other features so that the selection must be based on the pair of taus. There is a very large di-jet background from QCD events which must be overcome and the events require a trigger. Events are selected by requiring an isolated electron or muon with $P_{T}>25 \mathrm{GeV}$ and $|\eta|<2.5$ and hadronic jet with $E_{T}>30 \mathrm{GeV},|\eta|<2.5$, and a number of associated reconstructed tracks $N_{\text {track }}=1$ or 3 , The jet is also required to be narrow, having its energy deposits in the electromagnetic calorimeter confined with a cone of radius $R_{e m}=0.07$ in $\eta-\phi$ space. Figure 1 shows the reconstructed $Z$ mass with the peak at the correct value. The figure shows the mass resolution of $\sim 10 \%$ which is dominated by the missing $E_{T}$ resolution of the detector. The small residual QCD background is also shown. The subset of events with $N_{\text {track }}=3$ can be used to determine the tau decay vertex for the lifetime measurement discussed above.

\section{NEUTRAL HIGGS SEARCH}

The decay $H \rightarrow \tau \tau$ can be reconstructed using the technique described in the previous section and the mass of the Higgs measured. The production rates and branching ratios of the standard model higgs are too small for the signal to be seen above the QCD background using the dominant production process $g g \rightarrow H$. However the lower rate process $q q \rightarrow q q H \rightarrow q q \tau \tau$ while it may not enable a discovery to be made, will give information on the Higgs couplings. The two jets arising from the quarks in the production process are at large rapidity and can be used to reject background. Final states $\tau \tau \rightarrow \ell^{ \pm} h^{\mp} \ell_{p_{T}}$ and $\tau \tau \rightarrow \rightarrow e^{ \pm} \mu^{\mp}$ are used and the $\tau \tau$ invariant mass reconstructed as above with a resolution $\sigma \sim 0.1 M$. The table shows the expected signal and background [8]. It is important to emphasize that this result is based on a parametrized simu-

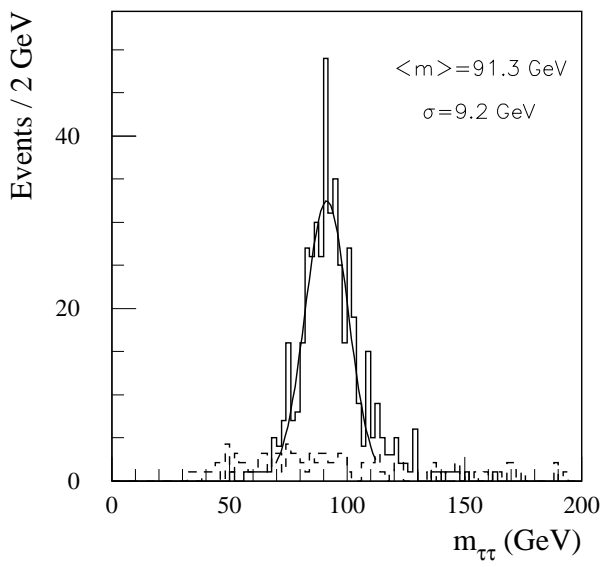

Figure 1. Reconstructed mass of the $Z \rightarrow \tau \tau$ signal for $30 \mathrm{fb}^{-1}$. The dashed line shows the background. From [1], figure 9.54

Table 1

Number of expected signal and background events for the $q q \rightarrow q q H \rightarrow \tau \tau j j$ channel, for $100 \mathrm{fb}^{-1}$ and two detectors. Cross sections are added for $\tau \tau \rightarrow \ell^{ \pm} h^{\mp} \ell_{T}$ and $\tau \tau \rightarrow e^{ \pm} \mu^{\mp}$

\begin{tabular}{c|cccccc}
$m_{H}$ & 100 & 110 & 120 & 130 & 140 & 150 \\
\hline Signal & 211 & 197 & 169 & 128 & 79 & 38 \\
Background & 305 & 127 & 51 & 32 & 27 & 24
\end{tabular}

lation that assumes that the tau tagging and jet tagging is not degraded in the presence of pile-up. Nevertheless it indicates a viable signal for mass range $110 \mathrm{GeV}<M_{H}<150 \mathrm{GeV}$ if the Higgs mass were already known from another process. Note that signal to background ratio is such that structure would clearly be seen at the Higgs mass.

In extensions to the standard model more higgs bosons are expected. The minimal supersymmetric model (MSSM) has three neutral $(h, A, H)$ and one charged $\left(H^{ \pm}\right)$particles. Their properties are determined by two parameters which can be taken to be the mass of $A$ and $\tan \beta$, which 
controls the coupling to tau's. $A$ and $H$ can be observed over certain regions of parameter space. The technique adopted is the same as that used for $Z \rightarrow \tau \tau$. Figure 2 shows three possible signals and the background which arises from QCD jets. This signal covers a large fraction of MSSM parameter space and is one of the most important probes of the MSSM Higgs sector.

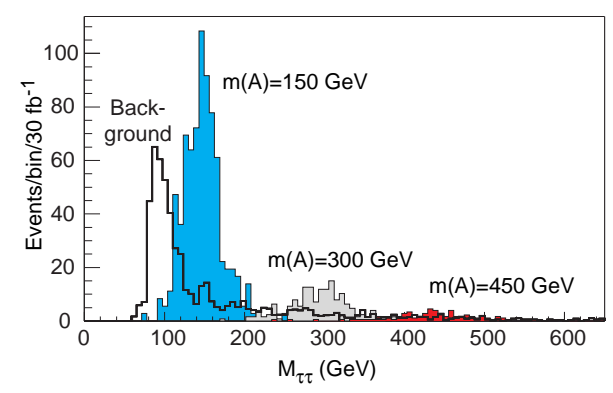

Figure 2. Reconstructed mass of the $A \rightarrow \tau \tau$ signal for $30 \mathrm{fb}^{-1}$ showing three possible masses. The background is shown as the unfilled histogram. From [1].

\section{CHARGED HIGGS}

A charged Higgs boson is expected to decay $H^{+} \rightarrow \tau \nu$ or $H^{+} \rightarrow q \bar{q}$. The latter decay involves the reconstruction of jets and the QCD background in the di-jet mass distribution is a serious difficulty to observation of this mode. The former decay is more promising. Production of a heavy $H^{ \pm}$via $q \bar{q} \rightarrow H^{+} H^{-}$is too small, the production via $g b \rightarrow H^{-} t$ has $\sigma\left(H^{+} t\right) \sim 1$ $\mathrm{pb}$ for a mass of $H^{ \pm}$of $400 \mathrm{GeV}$. The decay $H^{+} \rightarrow \tau \nu$ will result in either an isolated lepton or jet. The former is not useful so the latter is exploited. The final state $H t \rightarrow \tau \nu W b$ is identified by requiring three jets one of which is tagged as containing a b-hadron from the vertex system and requiring that these three jets are consistent with $t \rightarrow b W \rightarrow b q \bar{q}\left(m(j j)=M_{W} \pm 25\right.$ and $\left.m(j j b)=M_{t} \pm 25\right)$. Another jet with only one associated charged track and $p_{T}>100 \mathrm{GeV}$ is required along with missing $E_{T}(>100 \mathrm{GeV})$. The signal is in transverse mass of " $\tau$ " and missing $E_{T}$ which is shown in Figure 3. The peak is displaced below the Higgs mass as the missing $E_{T}$ has contributions from both neutrinos in the decay $H^{+} \rightarrow \tau \nu \rightarrow \pi \nu \nu$ and these tend to cancel.

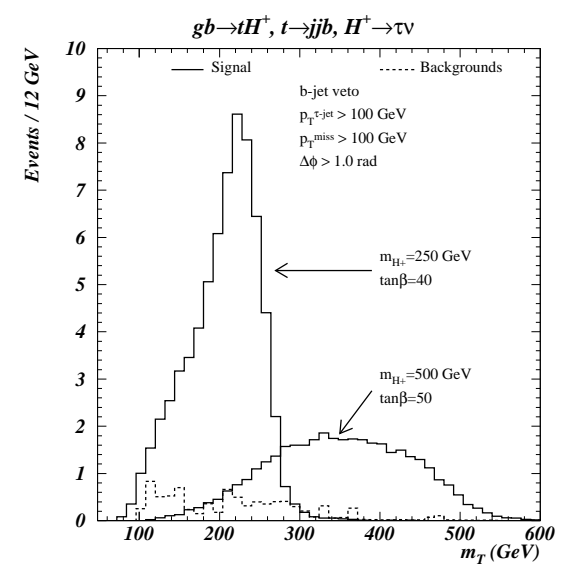

Figure 3. Transverse mass of the Missing $E_{T}$ and the jet identified as a tau. The signal from $H \rightarrow$ $\tau \nu$ shows two possible masses. The background is also shown. From [10].

\section{TAUS IN SUPERSYMMETRY EVENTS}

If supersymmetry (SUSY) is relevant to the problems of electroweak symmetry breaking, SUSY particles will be sufficiently light that the LHC will be a SUSY factory. Third generation sparticles are particularly interesting as they carry information about how supersymmetry is broken. In the case of the first two generations the mixing between the supersymmetric partners of the left and right handed charged leptons is expected to be small. However the two $\tilde{\tau}$ mass eigenstates are mixtures of $\tilde{\tau}_{L}$ and $\tilde{\tau}_{R}$ and we need to measure masses and mixings. The direct 
production of $\tilde{\tau}$ via electroweak processes such as $q \bar{q} \rightarrow \tau^{+} \tau^{-}$is small. In many cases many more $\tilde{\tau}^{\prime}$ 's are produced from decays of strongly produced squarks and gluinos via processes such as $\tilde{q} \rightarrow \widetilde{\chi}_{2} q \rightarrow q \tilde{\tau}^{+} \tau^{-} \rightarrow q \widetilde{\chi}_{1}^{0} \tau^{+} \tau^{-}$where $\widetilde{\chi}_{2}$ and $\widetilde{\chi}_{1}^{0}$ are weak gauginos and the latter is stable and exits the detector contributing to missing $E_{T}$. It is possible that taus may be the only leptons produced in gaugino decay.

Leptonic tau decays are of limited use as the origin of the lepton is unknown; one must search for hadronic tau decays that give rise to characteristic jets. SUSY events are expected to be very complex and one can rely on jet and $\mathbb{E}_{T}$ cuts to get rid of standard model background and obtain clean SUSY sample. This sample can then be studied in detail. The background to hadronic tau decays then arises from QCD jets in the SUSY event and a rejection factor of order 10 (the number of jets in a such an event) rather than order 100 which is needed in the analysis described in section 1 .

A particular model is used in the example illustrated here. ([1] vol II p. and [11]). Events are selected as follows: $\geq 4$ jets, one of which has $p_{t}>100 \mathrm{GeV}$ and the rest have $p_{t}>50$ $\mathrm{GeV}$, there are no isolated electrons or muons with $p_{t}>10 \mathrm{GeV}$ and $\mathbb{E}_{T}>100 \mathrm{GeV}$. The jets are examined and "tau" candidates extracted by selecting jets with low track multiplicity and invariant mass [12] using an algorithm based on a study using $Z$ decays to generate taus in the appropriate kinematic regime. The invariant mass of observed "tau" pairs is then shown in Figure 4 . A clear peak can be seen above the background which is dominated by events where one of the tau jet candidates is a misidentified QCD jet. If all the tau energy had been detected, the distribution would have had a sharp cut-off at

$$
M_{\tau \tau}^{\max }=M_{\tilde{\chi}_{2}^{0}} \sqrt{1-\frac{M_{\tilde{\tau}_{1}}^{2}}{M_{\tilde{\chi}_{2}^{0}}^{2}}} \sqrt{1-\frac{M_{\tilde{\chi}_{1}^{0}}^{2}}{M_{\tilde{\tau}_{1}}^{2}}}
$$

which evaluates to $61 \mathrm{GeV}$ in this example. The peak is below this due to the energy carried of by neutrinos in the tau decays.

The situation is rather more complicated than this analysis shows. The energy distribution of

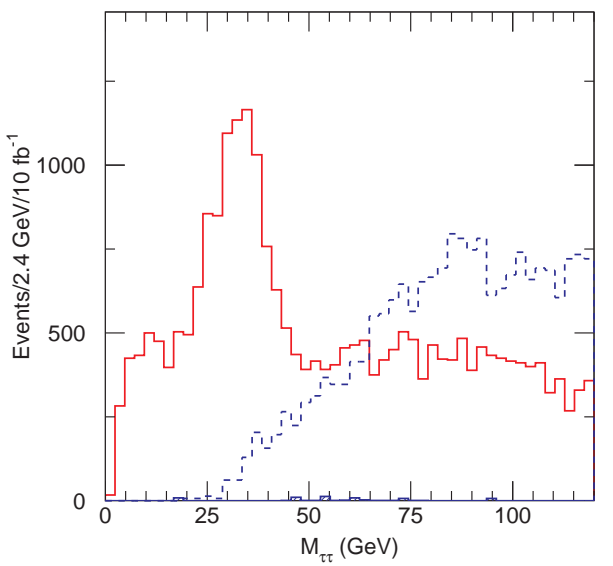

Figure 4. Invariant mass of the two tau candidates in SUSY events; the solid histogram shows the signal while the dashed shows the background from the combination of real tau and misidentified QCD jet, the small solid shows the background from QCD jets were both are misidentified. [1], Figure 20-42.

the visible tau decay products depends on the tau polarization and the dependence is largest in the $\tau \rightarrow \pi \nu$ final state and smallest in the states with large hadron multiplicity. A separation of these states could therefore yield information on the polarization of the taus. This in turn gives information on the $\tilde{\tau}$ composition. Simulation work on this difficult topic is underway.

\section{REFERENCES}

1. ATLAS Collaboration, "ATLAS Physics and Detector Performance Technical Design Report," LHCC 99-14/15.

2. D. Cavalli and B. Osculati, Atlas physics note ATL-PHYS-2000-014 (2000)

3. K. Kodama et al. [Fermilab E653 Collaboration], Phys. Lett. B 303, 359 (1993).

4. J. Ellis, M. E. Gomez, G. K. Leontaris, S. Lola and D. V. Nanopoulos, hep-ph/9911459.

5. J. L. Feng, Y. Nir and Y. Shadmi, Phys. Rev. D61, 113005 (2000).

6. J. Hisano, T. Moroi, K. Tobe and M. Yam- 
aguchi, Phys. Rev. D53, 2442 (1996) hep$\mathrm{ph} / 9510309$.

7. L. Serin and R. Stroynowski, Atlas physics note, ATL-PHYS-97-114 (1997).

8. A. Djouadi et al., arXiv:hep-ph/0002258.

9. D. Cavalli and S. Resconi, Atlas physics note, ATL-PHYS-2000-005 (2000)

10. K. A. Assamagan, Y. Coadou and A. Deandrea, Eur. Phys. J. directC 9, 1 (2002) arXiv:hep-ph/0203121.

11. I. Hinchliffe and F. E. Paige, Phys. Rev. D 61, 095011 (2000) arXiv:hep-ph/9907519.

12. Y. Coadou et al., ATLAS Internal Note ATLPHYS-98-126. 